Trascender, Contabilidad y Gestión. Vol. 6, Núm. 18 (septiembre - diciembre del 2021). Universidad de Sonora. Departamento de Contabilidad. México.

ISSN: 2448-6388. Reserva de Derechos 04-2015-04172070800-203.

\title{
La evaluación del desempeño, una nueva herramienta de la cultura de la transparencia aplicada al sector público en México
}

\author{
Performance evaluation, a new tool for the culture of transparency applied to the public \\ sector in Mexico
}

\author{
Luis Carlos Rodríguez Montaño ${ }^{1}$; Arturo Ordaz Álvarez ${ }^{2}$
}

Recibido: 6 de abril de 2021.

Aceptado: 15 de junio de 2021.

DOI: https://doi.org/10.36791/tcg.v0i18.126

JEL: H83. Administración Pública, Sector público.

\section{Resumen}

En el presente trabajo se describe y analiza el contexto general de la función de la evaluación del desempeño. Primero, se analizan las ideas generales del concepto. Posteriormente, se revisa su origen en las organizaciones de orden privado, y más adelante se describe y analiza cómo ha sido a través del tiempo su incorporación en el sector público en México vista como una herramienta de medición innovadora en el logro de sus objetivos. Sin embargo, la evaluación del desempeño aplicada en el sector público puede ser vista desde dos perspectivas, para algunos puede ser un área de oportunidad en cuanto al mejoramiento de la función del gobierno, para otros puede percibirse como una especie de castigo el cual cada vez más va tomando cauces institucionales y se va insertando en el quehacer gubernamental como medida de eficiencia.

Palabras clave: evaluación del desempeño, sector público, sector privado, gobierno.

\footnotetext{
${ }^{1}$ Luis Carlos Rodríguez Montaño. Licenciado en Administración Pública. Maestro en Ciencias Sociales especialidad de Políticas Públicas. Candidato a Doctor en Teoría y Análisis de Asuntos Públicos por el Colegio de Sonora y Candidato a Doctor en Administración Pública por el Instituto Sonorense de Administración Pública, A. C. Jefe del Departamento de Evaluación del Desempeño Legislativo del H. Congreso del Estado de Sonora. Correo: luiscarlosweb@hotmail.com

2 Arturo Ordaz Álvarez. Licenciado en Economía. Licenciado en Administración Pública. Licenciado en Artes Plásticas. Maestro en Administración. Doctor en Ciencias Sociales. Servidor público del Instituto Nacional del Consumidor, Secretaría de Educación Pública en Sonora, Centro Estatal de Estudios Municipales y el Instituto de Crédito Educativo del Estado de Sonora. Académico de la Universidad Kino, Maestría en Administración de la Universidad de Sonora, Maestría de Administración Pública del Instituto Sonorense de Administración Pública, A. C. y Doctorado en Administración Educativa del Instituto Pedagógico de Posgrado de Sonora, A. C. Correo: aordaz@sociales.uson.mx
} 


\begin{abstract}
This paper describes and analyzes the general context of the performance evaluation function. First, the general ideas of the concept are analyzed. Subsequently, its origin in private organizations is reviewed, and later it is described and analyzed how its incorporation into the public sector in Mexico has been over time, seen as an innovative measurement tool in the achievement of its objectives. However, performance evaluation applied in the public sector can be seen from two perspectives, for some it can be an area of opportunity in terms of improving the role of the government, for others it can be perceived as a kind of punishment which each time more is taking institutional channels and is being inserted in the governmental work as a measure of efficiency.
\end{abstract}

Keywords: performance evaluation, public sector, private sector, government.

\section{Introducción}

El siguiente trabajo presenta y contextualiza el propósito de la aplicación de la evaluación del desempeño para comprender su aplicación y su evolución como una herramienta que permite medir el cumplimiento de logros y objetivos en las organizaciones. El interés del presente trabajo reside en la idea que, la evaluación del desempeño ha evolucionado en su aplicación generalmente en las organizaciones de carácter privado, lo cual les permite hacer un diagnóstico del entorno para ser más eficaces en cuanto a la capacidad de ser competitivos frente a otras organizaciones. Parte del interés del presente ensayo es conocer cómo se dio la incorporación de la evaluación del desempeño en el sector público, es decir, cuáles fueron los canales de apertura que permitieron que dichas herramientas hicieran su aparición en el área gubernamental en México

Las causas por las cuales el sector público comienza a adoptar sistemas de medición subyacen principalmente por la desconfianza que tenían los ciudadanos en cuanto a que el gobierno no realizaba bien su trabajo, o simplemente no sabían que estaban haciendo. La auto implantación de los mismos entes gubernamentales de los sistemas de evaluación del desempeño, fueron una especie de medida para intentar recobrar la confianza de la ciudadanía sobre el trabajo que realizan los gobiernos. En el caso de la percepción ciudadana de la labor de los congresos, las encuestas colocaban al gobierno federal y a los gobiernos de los estados como organizaciones más bien opacas y con el menor rendimiento en cuanto a la eficacia en la implementación de sus programas públicos.

La evaluación del desempeño se incrustó en el gobierno mexicano como una función innovadora que abonaría a la cultura de la transparencia con el objeto de conseguir mejores niveles de aceptación por parte de la ciudadanía. Sin embargo, esta transición de adoptar ciertos modelos de evaluación que trajo consigo la ola democratizadora, no fueron implantados de golpe en México, sino que se fueron sembrando por sectores de la administración pública, con el fin de que el gobierno ofrezca mejores resultados de su gestión. 


\section{La evaluación del desempeño}

La evaluación del desempeño, de manera general, se ha convertido en la conjunción de dos palabras que interactúan de manera simbiótica, las cuales se utilizan casi de manera obligada en el lenguaje de las áreas de recursos humanos de las organizaciones. De acuerdo con la Real Academia Española (RAE, s.f.) la palabra evaluar se emplea como un verbo transitivo que le atribuye valor a algo o alguien, teniendo en cuenta diversos elementos o juicios, lo que se puede traducir en la acción de estimar o calcular el valor de algo, como pueden ser los conocimientos, las aptitudes o el rendimiento de los seres humanos. Por su parte, la palabra desempeño, de acuerdo con la misma RAE, se define como la acción de actuar, trabajar o dedicarse a una actividad. Derivado de lo anterior, se puede decir que la evaluación del desempeño, en general, comprende la acción de estimar o calcular el valor de algo o de alguien, en función de la realización de un trabajo o una actividad dada.

Algunas definiciones propuestas por diversos autores sobre la evaluación del desempeño la consideran un proceso que mide el desempeño del empleado, esto como el grado que una persona cumple con los requisitos de su trabajo, como es la idea de Milkovich y Boudreau (1994). Para Zerilli (1973), se trata de una apreciación sistemática del valor que un individuo demuestra por sus características personales y/o presentación con respecto a la organización de la que forma parte, expresado periódicamente conforme a un preciso procedimiento por una o más personas encargadas que conozcan al individuo y su trabajo.

Por lo general, en una evaluación, existe una parte subjetiva presente, o apreciación personal, ya que siempre habrá un evaluador que emite juicios de valor que también, a su vez, se le está evaluando su trabajo como evaluador.
Para Werther y Davis (1992), la evaluación del desempeño consiste en el proceso mediante el cual las organizaciones estiman el rendimiento global de los trabajadores. En tanto que Chiavenato (2000) la concibe como un sistema de apreciación del desempeño del individuo en el cargo y de su potencial de desarrollo. Uno de los conceptos más asequibles en cuanto a la simplificación y a los elementos que contiene se observa en Cuesta Santos (1999), que considera que la evaluación del desempeño consiste en un procedimiento que pretende valorar, de la forma más sistemática y objetiva posible el rendimiento de los trabajadores en una organización, y éste se realiza sobre la base del trabajo desarrollado, los objetivos fijados, las responsabilidades asumidas junto a las condiciones de trabajo y las características personales.

En un contexto evaluativo, se espera que con la aplicación de diversos tipos de indicadores se garantice la búsqueda de objetividad en el desempeño de los actores. En ese sentido, si un programa de evaluación del desempeño está bien planteado desarrollado y evaluado, generalmente trae beneficios a corto, mediano y largo plazo, en este escenario los beneficiarios son el evaluado, el jefe, la organización y la comunidad (Chiavenato, 2000).

Se puede afirmar, como lo advirtió French (1991), que en términos conceptuales la evaluación del desempeño es uno de los mecanismos para darle seguimiento al trabajo de las personas dentro de una organización, en donde el desempeño puede tener diversas acepciones como el nivel de rendimiento, aportaciones personales, productividad individual, observancia de normas y políticas, y otros atributos que puede contener un trabajo individual o de grupo de conformidad con estándares establecidos para un puesto. 


\section{Evolución de la evaluación del desempeño}

La evaluación del desempeño ha mostrado una evolución y ha tenido cambios significativos respecto de las ideas originales que se acuñaron a inicios del siglo XX. El precedente de la evaluación del desempeño se centraba en los resultados y no en los procesos, esto motivaba que el resultado final establecía el parámetro de la evaluación al final del trabajo. Hoy en día la evaluación ha mostrado avances en cuanto a las partes que conforman un proceso antes de obtener un resultado.

La evolución de la evaluación del desempeño es posible advertirlo en cuanto a que se gestó una transición en la manera de aplicar mejoras en una organización, en los cuales las organizaciones entendían los errores u omisiones del pasado, cambiando el paradigma de la evaluación tradicional con nuevas ideas de la evaluación moderna aplicadas de manera general o particular en las organizaciones. La evaluación del desempeño se presenta como el cambio entre evaluar los procesos y no solo el producto. De acuerdo a los sistemas verticales de vigilancia existía una respuesta pasiva-reproductiva del trabajador ante los deberes, y actualmente se busca que la evaluación sea de construcción activa, basada en incentivos y respuesta.

El rompimiento en la práctica también se ha dado en relación con las habilidades de los individuos, ya que antes se consideraba que éstas se mostraban de manera aislada, al evolucionar se busca la integración de las mismas habilidades aplicadas de acuerdo con los requerimientos de la organización. Finalmente, esta evolución de la evaluación, que otrora se daba de manera vertical y con poca delegación de responsabilidades, en la actualidad los esquemas de evolución sugieren que la evaluación debe de ser compartida, de corte más horizontal y estableciendo responsables en cada parte del proceso. Los procesos de evaluación aplicados en las organizaciones en ocasiones eran vistos como una especie de castigo hacia los trabajadores; y éste no se apreciaba como un proceso de aprendizaje o de adquisición de habilidades para mejorar el desempeño de los trabajos de la organización.

Hoy en día los procesos de evaluación del desempeño establecen estándares de calidad en la manera de realizar las funciones y procedimientos. También se da una evaluación del trabajador hacia arriba, se podría decir que la evaluación ha mostrado cambios significativos en cuanto a quienes va dirigida la evaluación. La evaluación del desempeño surge como una necesidad de mejora en las actividades que se realizan en un trabajo o encargo.

Poco a poco, las técnicas de evaluación se han ido adaptando a las necesidades de las organizaciones en el transcurso del tiempo y adaptándose a diversos sistemas de producción, podemos afirmar que cualquier sistema de evaluación del desempeño, persigue, en primera instancia, lograr medir y mejorar o alcanzar niveles de eficiencia en las diversas tareas que las organizaciones asignan a sus recursos humanos en cualquier organización.

\section{¿Por qué evaluar el desempeño en las organizaciones?}

Existen tres razones por las cuales se debe de evaluar el desempeño de los trabajadores (Dessler y Varela, 2011), la primera es que las evaluaciones nos ofrecen información relevante sobre la cual los supervisores están en mejores condiciones para la toma de decisiones 
al momento de otorgar promociones o mejoras salariales. La segunda es porque a través de las evaluaciones se puede establecer un plan de corrección de deficiencias de los procesos productivos, los cuales se descubren durante la evaluación, lo que se conoce como áreas de oportunidad. Finalmente, una evaluación del desempeño brinda la oportunidad de hacer revisiones de los planes trazados en la agenda que descubra las fortalezas y debilidades de los empleados.

La evaluación del desempeño bien aplicada en las organizaciones ayuda a identificar el talento de los recursos humanos con los que cuenta la empresa, y a partir de los resultados se establecen recompensas adecuadas evitando la subjetividad. Por otra parte, el trabajador sabe cuáles son los estándares que se esperan de su trabajo, y de qué manera al realizarlo, cuál será el impacto que tendrá al interior y hacia afuera de la organización.

La evaluación del desempeño permite establecer objetivos claros del lugar que se ocupa en ese momento, y en prospectiva, cuál es el lugar que se desea lograr con la consecución de los objetivos trazados. Otro de los aspectos importantes de por qué se debe evaluar el desempeño, es que supone que, en el logro de los objetivos personales, impacta en la formación y profesionalización del trabajo; en teoría eso provoca la motivación del trabajador y ofrece expectativas de aumento de sueldo, promociones, recompensas, etc.
A contrario sensu, si los objetivos no se están cumpliendo, la evaluación indica el camino que se debe seguir al detectar y corregir los errores y restablece los objetivos de la organización, o se valora la posición del empleado en ese puesto en caso de no estar a la altura de los objetivos que se pretenden lograr.

\section{Métodos de la evaluación del desempeño}

Los métodos de evaluación del desempeño que se aplican en las organizaciones pueden ser variados, generalmente la metodología es adaptativa al giro $\mathrm{y}$ objeto de cada organización y también existen métodos generales de carácter universal que se pueden aplicar a todo tipo de empresa. Dessler y Varela (2011) identifican algunos de los métodos más usados en las organizaciones:

a. Método de escala de puntuación gráfica. Este método es el más simple de los utilizados para realizar evaluaciones al desempeño sobre el personal. Se muestra en una escala que enumera una serie de características deseables y se utiliza un rango de desempeño para cada variable, los reactivos pueden ser grados de calidad o confiabilidad, o, de satisfactorio hasta insatisfactorio para cada indicador. Simplemente se asigna un valor de la escala a cada indicador que describa de la mejor manera el desempeño y al final se realiza la sumatoria de cada reactivo o variable (Tabla 2). 
Tabla 2

Método de escala de puntuación gráfica

\begin{tabular}{lllllll}
\hline Factores de evaluación & \multicolumn{5}{c}{ Niveles de puntuación } & Total \\
\hline Puntualidad & 1 & 2 & 3 & 4 & 5 & Sumatoria \\
\hline Trabajo en equipo & 1 & 2 & 3 & 4 & 5 & \\
\hline $\begin{array}{l}\text { Respeto a sus } \\
\text { compañeros }\end{array}$ & 1 & 2 & 3 & 4 & 5 & \\
\hline $\begin{array}{l}\text { Interés por aprender } \\
\text { Habilidades en el }\end{array}$ & 1 & 2 & 3 & 4 & 5 & \\
trabajo & 1 & 2 & 3 & 4 & 5 & \\
\hline Hace tareas extras & 1 & 2 & 3 & 4 & 5 & \\
\hline
\end{tabular}

Fuente: Elaboración propia.

b. Método de clasificación alterna. Este método clasifica a los trabajadores del mejor al peor apuntando a ciertas características. Es un sistema muy usado ya que resulta más fácil identificar el rendimiento malo y bueno de los trabajadores de una organización que pueda clasificar el rendimiento del empleado. De esta forma se identifica al empleado en el rango deseado y cuál está más bajo, se alternan los resultados y se clasifica al final.

c. Método de comparación por pares. En este tipo de evaluación cada trabajador se compara con todos los demás empleados en cada una de las características. Para este método, se utiliza una gráfica o tabla que muestra por pares a los empleados, y se añaden los rasgos a evaluar, para cada rasgo, el evaluador indica con signos de más (+) o menos (-) para identificar al mejor empleador de cada par. Posteriormente, se suma el número de veces que un trabajador califica como el mejor para cada rasgo, al final se evalúa por categoría de mejor o el menos apto.

Los métodos de evaluación en las organizaciones privadas son muy variados, y generalmente se diseña una metodología que combina los aspectos cualitativos del empleado y se utilizan diversas escalas con resultados cuantitativos para hacer más fácil realizar una evaluación basada en porcentajes o números absolutos que indican que un trabajador muestra un mejor desempeño de el mismo respecto de una situación anterior, o, muestra de entre los empleados quien puede cumplir mejor las tareas de la organización de acuerdo a los resultados mostrados. 
Hasta aquí, se pueden identificar de manera clara que las evaluaciones a las que hemos hecho referencia son del ámbito empresarial privado, lo que podría decirnos que este tipo de evaluaciones se enfoca generalmente con un objetivo de mejorar la productividad del trabajador basado en un contexto estadístico o categórico el cual tiene como finalidad saber cuál empleado es el que rinde más, comparándose con el mismo o con el resto de los empleados.

La variante de la evaluación del desempeño se presenta cuando se pretenden insertar modelos de evaluación del desempeño en el sector público, entidades que generalmente, al menos en México, no eran susceptibles de este tipo de mediciones, salvo en algunas áreas como de la salud, seguridad, educación o de la economía, pero, no en todos los casos, el tipo y forma de evaluación no atendía generalmente a parámetros de calidad, sino más bien a informar o describir los números alcanzados en un periodo de tiempo que normalmente era de un año a otro.

\section{Evaluación del desempeño en el sector público y privado}

Las organizaciones en México se clasifican como públicas, privadas o mixtas dependiendo de su origen legal, así como de la aportación del capital con que cuentan para la consecución de sus objetivos. La empresa privada es una organización que incorpora los postulados básicos del capitalismo y se conforma de aportaciones de capital privado. El objetivo principal de la empresa pública es la de invertir dicho capital con el fin de obtener una ganancia que permita la sobrevivencia de la organización (Tabla 3).

\section{Tabla 3}

Características y objeto de las organizaciones públicas y privadas

\begin{tabular}{ll}
\hline \multicolumn{1}{c}{ Públicas } & \multicolumn{1}{c}{ Privadas } \\
\hline Su capital pertenece al Estado & El capital es propiedad del inversionista \\
\hline $\begin{array}{l}\text { Buscan satisfacer necesidades de carácter } \\
\text { social }\end{array}$ & $\begin{array}{l}\text { Buscan un beneficio económico } \\
\text { particular }\end{array}$ \\
\hline $\begin{array}{l}\text { Representa al sector público de la } \\
\text { economía }\end{array}$ & Maximizan beneficios a través de ventas \\
\hline $\begin{array}{l}\text { Es un medio del Estado para intervenir en } \\
\text { la economía }\end{array}$ & Creada por acuerdo entre particulares \\
\hline Creada por mandato legal & Eficiencia de gestión \\
\hline
\end{tabular}

Fuente: Elaboración propia. 
La empresa privada evalúa la competencia del entorno externo para aplicar el principio de racionalidad económica de tal forma que le permita maximizar sus beneficios. De igual forma, la toma de decisiones se basa en los riesgos que ofrece el mercado que demanda los objetivos de su producción. Por su naturaleza la empresa privada considera una prioridad la implementación de sistemas de evaluación del desempeño, ya que la competencia de otras empresas privadas obliga a mejorar sus sistemas de producción.

Históricamente, la gestión de las empresas privadas se asocia a mejores modelos de eficiencia respecto a la empresa pública, y las evaluaciones del desempeño que éstas consideran se enfocan en la formación y mejora de su capital humano, quienes intercambian su fuerza de trabajo por un salario en beneficio de la organización.

Por su parte, las organizaciones de carácter público se crean por un mandato legal de derecho público, a través de una ley o de un decreto que funda y justifica su existencia. La misma ley le dota de los capitales de origen estatal, los cuales provienen de las contribuciones que de manera legal cobra el estado a sus ciudadanos. Las empresas u organizaciones públicas se crean con el objeto de satisfacer las necesidades de la población sin que ello implique la obtención de una ganancia, y el objetivo del Estado al crear este tipo de organizaciones es promover e impulsar el desarrollo socioeconómico de un país.

Si bien la empresa pública no reviste ganancias de carácter particular, generalmente este tipo de organizaciones no cuentan con competencia, éstas deben de aplicar el principio de racionalidad económica ante las limitantes del presupuesto público anual. Las empresas públicas cubren principalmente el área de la prestación de servicios y de las obras de infraestructura social. Al igual que la empresa privada, el Estado contrata empleados, los cuales se consideran empleados públicos, o que prestan servicios en nombre del estado.

En un contexto en donde los procesos de evaluación en los ámbitos de la gestión pública se enfocan en ciertos sectores públicos, pero se olvida de otros, es una de las razones por la cual el ciudadano promedio lo percibe al gobierno como un ente oneroso y de exiguos resultados (Hernández Borbolla, 2016; Hernández, 2014; Valdés Zepeda, 2004; Martínez Huerta, 2014).

La evaluación del desempeño en México, a través de indicadores de medición es una práctica que se puede decir que está en una etapa de adolescencia. Si bien tampoco podemos decir que sea una práctica demasiado novedosa, pues ya son varios los esfuerzos hechos en el ámbito federal y en algunos estados desde hace al menos quince años.

\section{La evaluación del desempeño del sector público en México}

Gran parte de la historia gubernamental en México durante el siglo XX, y a principios de la década del 2000, no dan un punto de referencia relevante de las bondades de la aplicación de sistema de evaluación del sector público en México. El discurso de la acción pública se caracterizaba generalmente por lograr buenos niveles de legitimidad política y control social de la población a través de una retórica de carácter economicista y de la defensa de la justicia social. Podemos afirmar que los primeros intentos de instituir en el lenguaje mexicano una política de evaluación gubernamental se dio en el sexenio de Miguel de la Madrid Hurtado (1982-1988).

Este primer acercamiento a tomar medidas de evaluación en el sector público fue más bien un reclamo 
de la sociedad ante el dispendio y las malas prácticas administrativas que se habían sucedido en sexenios anteriores. La creación de la Secretaría de la Contraloría General de la Federación fue el primer mazo institucional autoimpuesto de parte del gobierno federal para establecer una política de control y seguimiento de los objetivos del gobierno en México (Mejía, 2005, 56).

El modelo administrativo, influenciado por la burocracia de Weber entró en crisis durante el sistema presidencial mexicano. Dicho modelo presentaba bajos niveles de eficacia con altos márgenes de discrecionalidad, opacidad y corrupción (Vilchis, 2009). La administración pública se había convertido en una estructura pesada, que se caracterizaba por su ineficiencia al no responder a las demandas de la sociedad (Cabrero,1995).

Al respecto Gorriti (2007) comenta que la necesidad de implantar sistemas de evaluación del desempeño en el sector público no está en discusión, lo ve de hecho como una medida inevitable, la cuestión es en cuanto a los tipos de modelos de evaluación que se quieran implementar. Desde su perspectiva, este tipo de acciones se deben de incorporar como practicas cotidianas las cuales tienen el propósito de la profesionalización de los servidores públicos, ya que, de no hacerlo, el cambio gradual se obstaculiza, y se pone a merced de los cambios cualitativos los cuales se manifiestan principalmente con los cambios políticos o de índole tecnológico.

Es en la administración de Vicente Fox Quesada (2000-2006), con la aprobación de la nueva ley de transparencia y acceso a la información pública que el concepto de evaluación gubernamental adquiere carta de residencia en lo que primero fue abrir la información pública a los ciudadanos, y después se comenzarían a retomar prácticas y modelos de evaluación principalmente de las teorías de la Nueva Gestión Pública (Cabrero, 2003), la cual buscaba en gran medida implantar sistemas gerenciales como una nueva filosofía de las acciones de gobierno.

El presidente Fox creó el Sistema de Metas Presidenciales (SIMEP) el cual lejos de ser un sistema de evaluación de desempeño del gobierno eficaz y funcional, fungía como una lista de buenos deseos de las metas y objetivos que se pretendía lograr en las dependencias del gobierno federal.

La realidad era que México mostraba una pronunciada debilidad institucional en materia de evaluación gubernamental, esto ante la falta de la normatividad que permitiera evaluar todos los procesos de la gestión pública. El foco de atención de la evaluación del gobierno se centraba en algunos programas sectoriales que se habían sucedido unos a otros como programas insignia de diferentes administraciones, pero que al final de cada sexenio las evaluaciones hechas incluso por el mismo gobierno demostraban que no se conocían las causas que permitieran establecer objetivos reales de una evaluación de la gestión pública.

En esa etapa embrionaria y de transición política en el país, la creación del Consejo Nacional de Evaluación de la Política de Desarrollo Social (2005), instancia que mide la pobreza en México, y evalúa programas y políticas sociales del Gobierno Federal (CONEVAL) y de la Secretaria de la Función Pública (SFP), se puede decir que comienza una etapa en la cual las evaluaciones gubernamentales comienzan a verse con más seriedad, por una parte el CONEVAL evaluaba todos los programas sociales, y la SFP evaluaba a los funcionarios que aspiraban a ocupar un cargo dentro de la administración pública federal.

La sociedad a través de diversos organismos empresariales y asociaciones civiles exigían que el 
sector público en México fuera evaluado en relación con sus actividades, y más importante evaluar los resultados de la gestión pública (Caiden y Caiden, 2001), de tal forma que, una evaluación institucional, dotara de mayores capacidades al mismo gobierno, lo que derivara en una mayor eficiencia y eficacia de la gestión pública.

Para ese momento la atención se centró en los controles de la acción de gobierno a través de un ejercicio del legislativo por medio de la fiscalización y la rendición de cuentas, en ese momento, la confianza ya estaba perdida en el gobierno, la evaluación del sector público se podía notar que era una necesidad non grata para el propio gobierno, pero que debía aceptarse como medida de legitimidad.

Uno de los primeros estudios formales de evaluación del desempeño en el sector público en México lo realizó la OCDE a finales de los años 70 del siglo XX. Dicho estudio se basó en analizar el desempeño presupuestal del país tomando en cuenta lo que establecía la Ley de Presupuesto, Contabilidad y Gasto Público Federal. Posteriormente durante la administración de Ernesto Zedillo (1994-2000) se instauró el Programa para la Modernización de la Administración Pública 1995-2001, con el propósito de establecer mecanismos de rendición de cuentas en el ejercicio del presupuesto, es en ese momento en donde podemos hablar de un modelo de sistema de evaluación del desempeño más funcional en el sector público (Pardo, 2008).

A partir de ese momento se concentran sistemas de evaluación del desempeño en varias áreas de la administración pública federal desde la óptica de la aplicación del presupuesto público, programas sociales como PROGRESA fueron objeto de evaluaciones sistemáticas, para posteriormente dar paso a los sistemas de evaluación de la nueva estructura programática tomando el enfoque de la planeación estratégica evaluando y dándole seguimiento al ejercicio del presupuesto público.

Podemos afirmar que la evaluación del desempeño en el sector público tiene su origen en México en los sistemas de control del proceso presupuestario, la evaluación del desempeño en el gobierno se dio de manera paralela a la evolución de los sistemas de presupuesto, al pasar de los sistemas tradicionales, al de presupuesto por programas y finalmente al presupuesto basado en resultados.

Es través de varias reformas a las leyes de presupuesto que poco a poco se comienzan a establecer parámetros de gestión por resultados, se comienzan a establecer metas y objetivos de cada uno de los programas del presupuesto de egresos que están vinculados al Plan Nacional de Desarrollo, y que consideraron un Programa Anual de Evaluación (PAE) y un Programa de Mejoramiento de la Gestión (PMG).

El sistema de evaluación del desempeño en el sector público en México, en sus inicios se vinculó de manera sustancial al proceso presupuestario, el inicio de dicho proceso comienza con la presentación del Plan Nacional de Desarrollo (PND), el cual establece objetivos generales a mediano plazo (6 años), estos objetivos se materializan en el diseño de un presupuesto público basado en resultados el cual define sus proyectos estratégicos a través de la metodología del marco lógico, el cual vincula los objetivos estratégicos con los programas sociales derivados del PND.

La última fase del sistema de evaluación del presupuesto se da con la revisión que hace la Auditoria Superior de la Federación (ASF) a los ingresos y al ejercicio del gasto público enfocando las auditorías a los programas y a la evaluación de las políticas públicas que tienen un impacto en el gasto público del gobierno como se puede apreciar en la imagen 1. 


\section{Imagen 1}

Sistema de evaluación de la gestión en el sector público en México

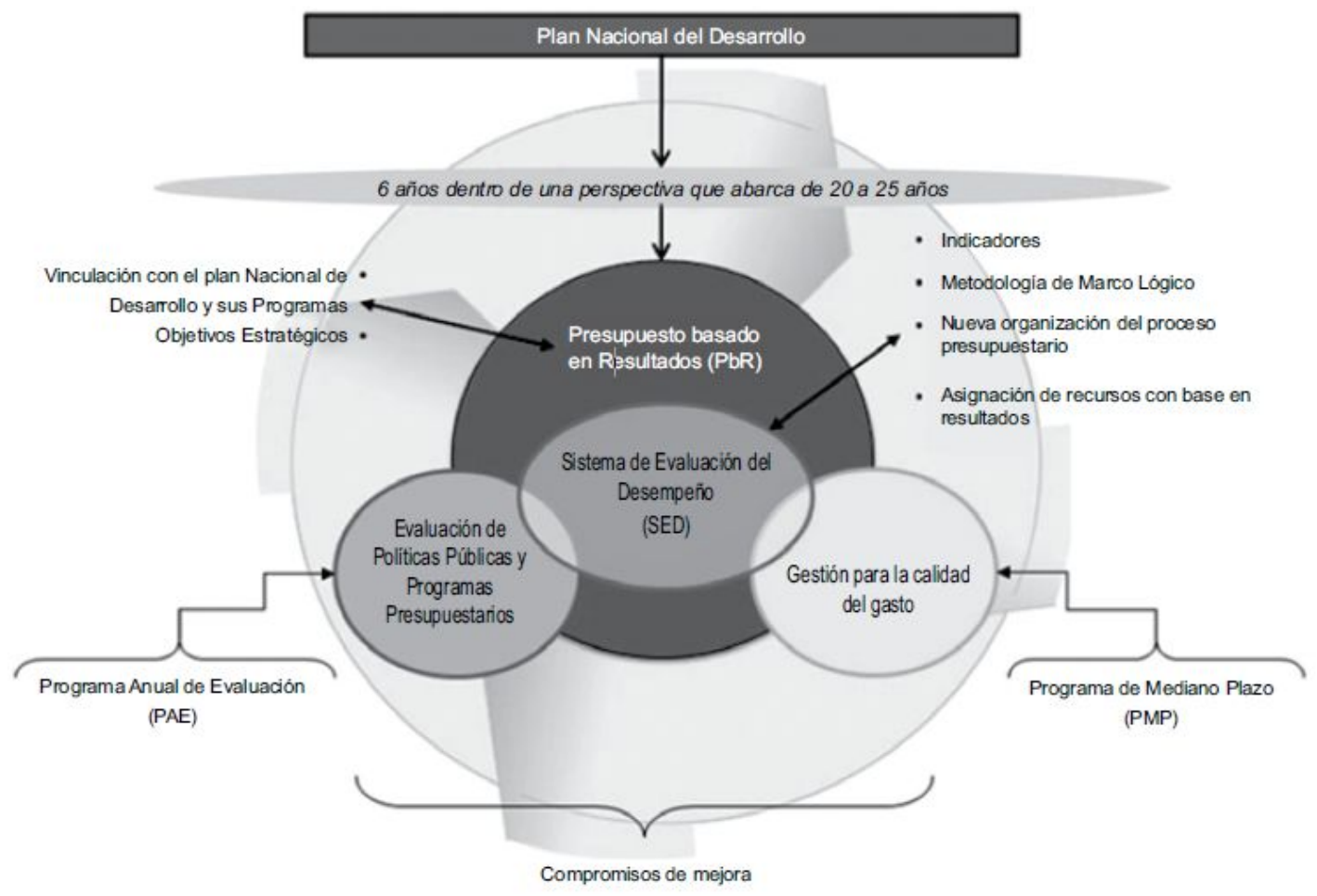

Fuente: SED, Secretaría de Hacienda y Crédito Público (s.f.).

Estos métodos de evaluación se han retomado por los estados en sus propios planes de desarrollo y en el diseño de sus presupuestos de egresos. A través de diversas reformas constitucionales, el congreso federal ha motivado la armonización de las leyes de los estados para que a través de la disciplina financiera se cumplan los objetivos de la gestión del gobierno y se aseguren finanzas sanas de la federación y de los estados, evaluando constantemente el diseño del presupuesto y el ejercicio del gasto público principalmente.

Hasta la fecha, la evaluación del desempeño del sector público aún mantiene su atención principalmente en los correspondiente al ejercicio del ingreso y del gasto público. Y esta situación no es del todo ajena, si tomamos en cuenta que unos de los principales problemas de nuestro país se concentran o se ha concentrado principalmente en la corrupción, el cual deriva de los malos manejos del presupuesto nacional y de los estados.

\section{Conclusiones}

El proceso de evaluación del desempeño ha transitado por un camino en el cual las ideas aplicadas a las organizaciones privadas en el ámbito de la evaluación permean en el sector público con el objeto de dar a 
conocer qué se hace y cómo se hace el trabajo en el sector público nacional y local.

Esta situación de adoptar modelos evaluativos en el sector público, se dio principalmente después de la entrada en vigor de las leyes de transparencia, las cuales, de manera tajante entraron en escena, para poner en un escaparate la función pública de diversos actores políticos que había estado en una zona de confort, como el caso del legislativo tanto a nivel nacional como en lo local, el cual había pasado desapercibido por la ciudadanía al no saber de manera clara el alcance de sus funciones.

Los sistemas de evaluación del desempeño en el sector público deben de transitar, de ser aplicativos a los procesos presupuestarios, a ser un parámetro de cómo ofrece el estado, en el orden federal y estatal, los servicios que la población espera de ellos, sin importar la base ideológica o partidista que llegue al poder, sino que, los sistemas de evaluación deben de instalarse en el marco normativo mexicano con una base constitucional, de tal forma que ante el cambio político no se afecte la normatividad que sea aplicable a quienes ostenten el poder en turno.

La evaluación del desempeño ya no debe de ser vista como un castigo para la toma de decisiones en la administración pública, sino más bien como una herramienta de apoyo, por medio de la cual se busque la concretización de los objetivos que se autoimpone el estado en los tres órdenes de gobierno. Quienes sirven en esas instancias de poder se deben a los electores y deben de responder a ellos, a la sociedad en general. En esta interrelación resulta relevante la institucionalización de canales de comunicación que propicien confianza mutua de las partes y alienten la vida democrática. En este sentido es que se comprende el proceso que se sigue en el caso del sector público respecto al proceso de la evaluación del desempeño de los servidores públicos o empleados del estado mexicano.

La razón de ser de las instancias públicas se encuentra en la concreción del interés público y el bienestar de la población. El servicio público a su cargo subsume la responsabilidad social que, como organismos sociales, propios del Estado debe cumplir para dar cumplimiento a sus obligaciones legales $\mathrm{y}$ al compromiso social $\mathrm{y}$ político con todos los sectores y miembros de la sociedad.

\section{Referencias}

Cabrero, E. (1995). Del administrador al gerente público. Instituto Nacional de Administración Pública. INAP. Universidad Autónoma del Estado de México UAEM.

Caiden, G., y Caiden N. Enfoques y lineamientos para el seguimiento, la medición y la evaluación del desempeño en programas del sector público. en Revista de Servicio Público, Año 52, No. 1, (eneromarzo, 2001).

Cuesta, S. A. (1999). Tecnología de gestión de recursos humanos. Ed. Academia.

Chiavenato, I. (2000). Administración de recursos humanos. McGraw-Hill.

Dessler, G., y Varela R. (2011). Administración de recursos humanos. Enfoque latinoamericano. Pearson Educación.

French, W. L. (1991). La administración de personal, desarrollo de recursos humanos. Ed. Noriega Limusa.

Gorriti, M. (2007). La Evaluación del Desempeño en las Administraciones Públicas Españolas. Revista de Psicología del Trabajo y de las Organizaciones, 23(3), 367-387. 
Hernández, A. (2014). Poder Legislativo caro y poco transparente. Integralia. En El Financiero. http://www.elfinanciero.com.mx/politica/caro-y-pocotransparente-el-poder-legislativo.html

Hernández Borbolla, M. (2016). Exceso de Comisiones y resultados paupérrimos en el Congreso. The Huffington Post. http://www.huffingtonpost.com.mx/

2016/10/03/exceso-de-comisiones-yresultadospauperrimos-en-el-congreso/

Martínez, D. (2014). Diputados federales ganan 74 salarios mínimos y no rinden cuentas. En Visión Legislativa. http://visionlegislativa.com/diputadosfederales-ganan-74-salarios-minimos-y-norindencuentas-sin-embargo-19ags14/

Mejía, J. (2005). La evaluación como herramienta para una gestión pública orientada a resultados. $\mathrm{La}$ práctica de la evaluación en el ámbito público mexicano. Caracas, CLAD. (Documentos Estudios de Casos, 2), 56. http://www.clad.org.ve

Milkovich, G., y Boudreau, J. (1994). Dirección y administración de Recursos Humanos. 384-421. Delaware; Addison-Wesley Iberoamericana; McGrawHill.
Pardo, M. (2008). La modernización administrativa de México 1940-2000. México, El Colegio de México.

Valdés, A. (2004). El Poder Legislativo en opinión de los mexicanos: Un análisis de la crisis de imagen. En Estudios Políticos, 42 (septiembre-diciembre), 33-59. http://www.revistas.unam.mx/index.php/rep/article/vie w/37628

Vilchis, J. (2009). La nueva gerencia pública en México: Una medición de su intensidad e impactos en las entidades del país. En Convergencia, 199-227. http://www.scielo.org.mx/scielo.php?script=sci_arttext \&pid=S1405-14352009000100008\&lng=es\&tlng=es

Werther, W. B., y Davis, K. (1992). Administración de Personal y Recursos Humanos. McGraw-Hill.

Zerilli, A. (1973). Valoración de personal. Deusto.

\section{Recursos de internet}

Real Academia Española. (s.f.). https://dle.rae.es/diccionario.

Secretaría de Hacienda y Crédito Público. (s.f.). Sistema de Evaluación del Desempeño.

https://www.apartados.hacienda.gob.mx/sed/html/docs/ DirectricesSED.html 